Z Gerontol Geriat 2021 · 54:633-635 https://doi.org/10.1007/s00391-021-01965-w Angenommen: 5. August 2021

(c) Springer Medizin Verlag $\mathrm{GmbH}$, ein Teil von Springer Nature 2021

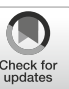

\section{Autoren}
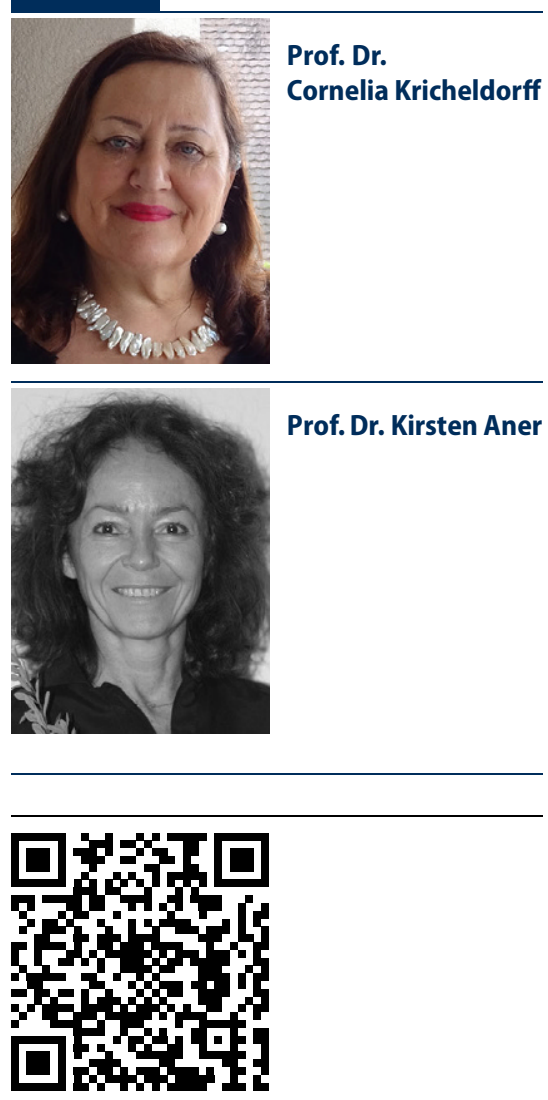

QR-Code scannen \&Beitrag online lesen

\title{
Facetten der Versorgungsrealität von Menschen mit Demenz
}

\author{
Cornelia Kricheldorff' $\cdot$ Kirsten Aner ${ }^{2}$ \\ 'Beratung - Prozessbegleitung - Training, Freiburg, Deutschland \\ ${ }^{2}$ Fachbereich Humanwissenschaften, Fachgebiet Lebenslagen und Altern, Universität Kassel, Kassel, \\ Deutschland
}

Die kontinuierlich zunehmende Zahl von Menschen mit Demenz rückt angesichts brüchiger werdender familialer Ressourcen die Frage der Versorgungsrealität immer stärker in den Blick von gerontologischer, geriatrischer und pflegewissenschaftlicher Forschung wie auch in den Fokus der sozialen Altenhilfe in den Kommunen [10]. Epidemiologische Befunde differieren zwar etwas, gehen aber im Mittel von aktuell 1,7 Mio. Menschen mit unterschiedlichen Formen der Demenz in Deutschland aus [3, 14]. Basierend auf verschiedenen Datenquellen zur Vorausberechnung der weiteren Bevölkerungsentwicklung wird sich diese Zahl bis zum Jahr 2050 auf 2,4-2,8 Mio. erhöhen [3, 4]. In dieser Hinsicht werden zukünftig auch verstärkt ältere Menschen mit Migrationshintergrund stärker zu berücksichtigen sein. Gegenwärtig sind in Deutschland ca. 137.000 Menschen mit Zuwanderungsgeschichte von einer Demenz betroffen, mit deutlich steigender Tendenz. Verantwortlich dafür sind u.a. ihre überproportional quantitative Zunahme und deutlich höhere Gesundheitsrisiken, verglichen mit Gruppen Gleichaltriger aus der Aufnahmegesellschaft $[2,8,11]$.

Gleichzeitig ist aufgrund demografischer Veränderungen und sich stark wandelnder familiärer Strukturen absehbar von einem Rückgang des Pflegepotenzials in den Familien auszugehen. Diese sind oft heute schon bis zu ihrem Limit belastet, weil sie die tägliche Sorge und Unterstützung für ihre pflegebedürftigen alten Angehörigen kaum noch mit beruflichen Aufgaben und eigenen familiären Verpflichtungen vereinbaren können $[1,5]$. In der Konsequenz führt das immer stärker zur Notwendigkeit, geeignete
Alternativen zu den eher traditionellen Pflegesettings im häuslichen Umfeld zu entwickeln, die aber heute noch die tragende Säule in der Versorgungsrealität für rund zwei Drittel aller Menschen mit Demenz in Deutschland darstellen.

Vor diesem Hintergrund hat sich in den vergangenen Jahren bereits ein breites Spektrum an alternativen Ansätzen, Versorgungs- und Wohnangeboten für pflegebedürftige Menschen entwickelt. Der Schwerpunkt liegt auf der Versorgung von Menschen mit Demenz in ambulanten Settings. Dieser Ausbau wurde vielfach auch ermöglicht durch neue Landesgesetze und Verordnungen, die die Logik der alten Heimgesetzgebung mittlerweile in fast allen Bundesländern ersetzen. Zusätzlich haben entsprechende Modellprogramme die Erprobung und Implementierung neuer Konzepte und Organisationsformen in der Pflege auch gezielt gefördert. In der Trägerlandschaft wird allerdings noch immer kontrovers diskutiert, ob und inwieweit sich diese neuen, eher kleinen und ins Quartier integrierten Versorgungsformen wirtschaftlich rechnen und mit ihrem Mix an Alltagsbetreuung den Bewohner*innen entsprechend mehr Lebensqualität bieten können. Unübersehbar ist aber, dass auf diesem Weg wichtige Diskurse zur Gestaltung von demenzfreundlichen Umwelten und die Entwicklungen eines demenzsensiblen Umgangs mit Betroffenen bis hinein in die Häuslichkeit [12] und die Akutkrankenhäuser [13] angestoßen worden sind.

Trotz der inzwischen stark ausgeprägten Diversität alternativer Konzepte zur Pflege und zur Betreuung von Menschen mit Demenz lassen sich doch einige ge- 
meinsame Prämissen identifizieren, die sich als fachliche Grundorientierungen beschreiben lassen. Eine davon ist das Bemühen um die Sicherung der personalen Identität, die auch eine Basisorientierung im Konzept der personenzentrierten Pflege von Tom Kitwood darstellt, das bereits Mitte der 1990-er Jahre entwickelt wurde. Verbunden mit der Methode des "dementia care mapping", wird dabei die Einzigartigkeit der Person in den Mittelpunkt gerückt [6]. Eine zweite konzeptionelle Grundorientierung neuer Versorgungskonzepte, die sich v.a. an Menschen mit Demenz richten, ist die Wahrung von Autonomie trotz Beeinträchtigung der Selbstständigkeit. Das bedeutet, dass Willensbekundungen von Betroffenen wahrgenommen, respektiert und geachtet werden, auch wenn dies aufgrund kognitiver Beeinträchtigungen z.T. über eine validierende Form der Kommunikation oder eine stellvertretende Wahrnehmung der Interessen von Betroffenen erfolgen muss [9]. Eine dritte gemeinsame Prämisse der fachlichen Ausrichtungen stellt die Stärkung sozialer Teilhabe dar, die in Verbindung mit sozialraumorientierten Konzeptionen für die Gewährleistung der sozialen Integration und Inklusion von Menschen mit Demenzen steht [7, S. 120].

Der verstehende und akzeptierende Umgang mit der stetig wachsenden Gruppe von Menschen mit Demenz muss also weit über die Sicherstellung von Pflege hinausgehen und als gesamtgesellschaftliche Aufgabe betrachtet werden, die in vielfältiger Weise unterschiedliche Bereiche von Sorge, Pflege und Behandlung tangiert. Deshalb nimmt der hier vorliegende Themenschwerpunkt sehr unterschiedliche Fragen zu aktuellen Versorgungsrealitäten in den Blick und das aus ganz verschiedenen disziplinären Perspektiven.

Mit den Einstellungen gegenüber Menschen mit Demenz beschäftigt sich der Beitrag von Andreas Huber und Alexander Seifert, beide Universität Zürich. Sie stellen die Ergebnisse einer schweizweiten telefonischen Bevölkerungsbefragung unter rund 860 Personen über 18 Jahre vor, die in der zweiten Jahreshälfte 2018 durchgeführt worden ist. Dabei zeigen sich sowohl das individuelle Erleben der Alters- phase im persönlichen Umfeld wie auch die Haltung zum eigenen Alter als signifikante Prädiktoren für die entsprechenden Einstellungen zu Menschen mit Demenz. Deutlich wird auch die Bedeutung des Wissens und der erlebten Kontakte zu betroffenen Personen. Diese Ergebnisse unterstreichen also sehr deutlich, dass die soziale Integration von Menschen mit Demenzen nicht zuletzt über alltägliche Begegnungen und das gemeinsame Erleben gelingt. Sozialraumorientierte Pflege und Versorgungskonzepte bieten dafür einen ermöglichenden Rahmen.

Mit der Perspektive der Vereinbarkeit von Pflege bei Demenz, Familie und Beruf beschäftigt sich der zweite Beitrag von Lydia Neubert, Sophie Gottschalk, HansHelmut König und Christian Brettschneider vom Institut für Gesundheitsökonomie und Versorgungsforschung am Universitätsklinikum Hamburg-Eppendorf. Präsentiert und beschrieben werden darin die zentralen Ergebnisse einer qualitativen Studie, basierend auf 14 narrativen Interviews mit pflegenden Angehörigen von Menschen mit Demenz. Diese wurden mittels der dokumentarischen Methode nach Nohl ausgewertet und mündeten in eine Typenbildung. Eine der zentralen Aussagen zielt auf die Bedeutung der parallel zur familiären Pflege ausgeübten beruflichen Tätigkeit, die einerseits eine zusätzliche Belastung, andererseits aber auch ein entlastender Faktor sein kann. Hilfreich dafür erwies sich eine beratende oder therapeutische Begleitung für berufstätige pflegende Angehörige - eine spezifische neue Facette in der Versorgungslandschaft, die zur Stabilisierung häuslicher Pflegsettings beitragen kann.

Mit der Situation von Menschen mit Demenz im Krankenhaus beschäftigen sich zwei weitere Beiträge des Themenschwerpunkts. Da ist zunächst die Arbeit von Jasmin M. Greskötter vom Department für Pflegewissenschaft der Universität Witten/ Herdecke. Sie richtet ihren Blick auf Angehörige von Patienten mit Demenz als Bindeglied und Vermittler im Akutkrankenhaus. Auf der Basis einer systematischen Literaturrecherche arbeitet sie förderliche und hinderliche Faktoren für die verschiedenen Rollen heraus, die die Angehörigen dabei einnehmen können - $u$. a. in den Rollen als Fürsprecher*innen, Expert*innen und Berater*innen. In der Arbeit werden auf der Basis des Reviews die Voraussetzungen und Bedingungen für das Gelingen dieser vermittelnden Aufgaben herausgearbeitet.

Über die Evaluation eines Trainingsprogramms für Pflegekräfte berichten im zweiten Beitrag zum Setting Akutkrankenhaus Thomas Johann Gehr, Cornel Christian Sieber, Ellen Freiberger und Sabine Alexandra Engel. Der Beitrag mit dem Titel „EduKation demenz ${ }^{\circledR}$ Nursing in the acute hospital setting. Evaluation of a dementia training program: a pilot study" zeigt die Auswirkungen eines zweitägigen Schulungsprogramms zum Thema Demenz, das mit einer deutlichen Komponente der Selbstreflexion verbunden ist. Dieses Vorgehen wird verglichen mit einer 1,5-stündigen Schulung, wie sie für Pflegekräfte im Akutkrankenhaus bislang eher der übliche Standard ist. Das evaluierte neue Schulungsprogramm wird im Ergebnis und unter Verweis auf relevante Outcomes als wirksame Unterstützung für Pflegekräfte beschrieben. Unter anderem konnten statistisch signifikante Verbesserungen des wahrgenommenen Belastungserlebens und eine verbesserte Handlungssicherheit in Bezug auf Patient*innen mit Demenzen aufgezeigt werden.

Der fünfte Beitrag nimmt die Forschungsperspektive der Forschenden selbst ein und geht der Frage nach, wie Forscher*innen aus dem ENSURE Consortium die Anwendung der Tools bewerten, die speziell zur Entscheidungsassistenz für die informierte Einwilligung zur Forschungsteilnahme von Menschen mit Demenz im Kontext der Forschungskooperation im Konsortium entwickelt worden sind. Im Beitrag von Theresa S. Wied, Aoife Poth, Johannes Pantel, Frank Oswald und Julia Haberstroh, alle Mitglieder des ENSURE Consortium, werden die Ergebnisse einer Onlineumfrage unter 19 Demenzforscher*innen aus Deutschland und Portugal vorgestellt. Der Titel lautet: „How do dementia researchers view support tools for informed consent procedures of persons with dementia?"

Zusammenfassend kann also festgehalten werden, dass der Themenschwerpunkt Facetten der Versorgungsrealität von Menschen mit Demenzen einen wei- 
ten Bogen schlägt, der auch den Blick für künftige Bedarfe schärft. Die 5 Beiträge, die einen breiten Range von wichtigen Ergebnissen skizzieren, werden die Fachdiskurse zur Zukunft der Pflege und zur Versorgung von Menschen mit Demenz in besonderer Weise sicher befruchten.

\section{Korrespondenzadresse}

\section{Prof. Dr. Cornelia Kricheldorff}

Beratung - Prozessbegleitung - Training Barbarastr. 7, 79106 Freiburg, Deutschland cornelia.kricheldorff@t-online.de

\section{Prof. Dr. Kirsten Aner}

Fachbereich Humanwissenschaften, Fachgebiet Lebenslagen und Altern, Universität Kassel

Arnold-Bode-Str. 10, 34127 Kassel, Deutschland aner@uni-kassel.de

Interessenkonflikt. C. Kricheldorff und K. Aner geben an, dass kein Interessenkonflikt besteht.

\section{Literatur}

1. Alltag S, Conrad I, Riedel-Heller StG (2019) Pflegebelastungen bei älteren Angehörigen von Demenzerkrankten und deren Einfluss auf die Lebensqualität. Z Gerontol Geriat 52:477-486

2. Bartig S, Rommel A, Wengler A, Santos-Hövener C, Lampert T, Ziese T (2019) Gesundheitsberichterstattung zu Menschen mit Migrationshintergrund - Auswahl und Definition von (Kern-)Indikatoren. J Health Monit 4(3):30-49. https://doi.org/10. 25646/6069

3. Deutsche Alzheimer Gesellschaft (2020) Die Häufigkeit von Demenzerkrankungen. Informationsblätter der Deutschen Alzheimer Gesellschaft. https://www.deutsche-alzheimer.de/fileadmin/ Alz/pdf/factsheets/infoblatt1_haeufigkeit_ demenzerkrankungen_dalzg.pdf.Zugegriffen:29. Juli 2021

4. Glaeske G (2020) Demenzreport 2020. Hrsg. Socium Forschungszentrum. Universität Bremen \& HKK. https://www.hkk.de/.../2020_hkk_ demenzreport-2020_web.ashx

5. JacobsK, Kuhlmey A, GreßS, Klauber J, Schwinger A (Hrsg) (2021) Pflege-Report 2021 Schwerpunkt: Sicherstellung der Pflege: Bedarfslagen und Angebotsstrukturen.Springer, Heidelberg

6. Kitwood T (2019) Demenz - Der person-zentrierte Ansatz im Umgang mit verwirrten Menschen, 8. Aufl. Hogrefe, Göttingen

7. Kremer-Preiß U, Maetzel J, Huschi G (2021) Neue Wohnformen für Pflegebedürftige - Mehrwert oder bloß Mehraufwand? In: Jacobs K, Kuhlmey A, Greß S, Klauber J, Schwinger A (Hrsg) (2021) Pflege-Report 2021 Schwerpunkt: Sicherstellung der Pflege: Bedarfslagen und Angebotsstrukturen. Springer, Heidelberg. S. 119-129

8. Monsees J, Hoffmann W, Thyrian JR (2019) Prävalenz von Demenz bei Menschen mit Migrationshintergrund in Deutschland. Z Gerontol Geriat 52:654-660
9. Ritzi S, Kruse A (2019) Würde, Freiheit, Leiblichkeit. ZGerontol Geriat 52:243-248

10. Rubin Y (2020) Kommunale Alten(hilfe-)planung. In: Aner K, Karl U (Hrsg) Handbuch Soziale Arbeit und Alter. Springer, Wiesbaden, S55-71

11. Schmachtenberg T, Monsees J, Hoffmann W, van den Berg N, Stentzel U, Thyrian JR (2020) Comparing national dementia plans and strategies in Europe-is there a focus of care for people with dementia from a migration background? BMC Public Health 20:784. https://doi.org/10.1186/ s12889-020-08938-5

12. Stemmer R, Gräßel E, Schmid M (2019) Einzelaktivierung von Menschen mit Demenz im häuslichen Setting.Z Gerontol Geriat 52:256-263

13. Teichmann B, Bauer JM, Beyreuther K, Kruse A (2019) Forschung zur Versorgung von Menschen mit Demenz im Akutkrankenhaus. Z Gerontol Geriat 52:208-211

14. Thyrian JR, Boekholt M, Hoffmann W et al (2020) Die Prävalenz an Demenz erkrankter Menschen in Deutschland - eine bundesweite Analyse auf Kreisebene. Nervenarzt 91:1058-1061. https:// doi.org/10.1007/s00115-020-00923-y 